\title{
Rotatividade docente na rede municipal de ensino do Rio de Janeiro
}

\author{
Marcela Brandão Cunha ${ }^{1}$ \\ ORCID: 0000-0002-8817-6902
}

\section{Resumo}

0 estudo tem como objetivo investigar as dinâmicas de rotatividade docente na rede municipal de ensino do Rio de Janeiro. Tem-se como pressuposto a existência de padrões não aleatórios de distribuição de professores entre escolas, destacando-se possíveis convergências entre perfis de professores e alunos, principalmente no que diz respeito a indicadores de qualificação docente e ao nível socioeconômico e desempenho escolar dos estudantes. Dessa forma, professores com características mais favoráveis tenderiam a lecionar em escolas com alunos de nível socioeconômico mais alto e melhor desempenho escolar, e vice-versa. Em relação à revisão de literatura, destacam-se estudos internacionais que investigam a mobilidade de professores dentro de uma mesma rede de ensino. A pesquisa em questão abrange os docentes que ingressaram na rede municipal de ensino do Rio de Janeiro entre 2002 e 2012 e investiga a relação entre a rotatividade docente nas escolas e as principais características de professores e alunos que as compõem. As análises são baseadas na legislação municipal acerca das transferências desses profissionais de ensino entre escolas; em dados funcionais dos educadores e registros de transferências de escola, fornecidos pela Secretaria Municipal de Educação do Rio de Janeiro (SMERJ); além de dados da Prova Brasil, referentes ao nível socioeconômico e desempenho escolar dos alunos. Dentre os principais resultados, é possível sugerir a possibilidade de convergência entre perfis menos favoráveis de professores e alunos em instituições de ensino com maior rotatividade docente.

\section{Palavras-chave}

Rotatividade docente - Distribuição não aleatória de professores entre escolas Convergência entre perfil de professores e alunos.

1- Instituto Federal de Educação Ciência e Tecnologia do Rio de Janeiro (IFRJ), Rio de Janeiro, RJ, Brasil. Contato: marcela.cunha@ifrj.edu.br. 


\section{Teacher's turnover in the Rio de Janeiro City Department of Education}

\section{Abstract}

The purpose of the study is to investigate the teachers 'turnover dynamics, in the Department of Education of the City of Rio de Janeiro. An existing non-random distribution pattern of teachers among schools was assumed, with a highlight on convergences, if any, between teachers and student profiles, especially those regarding teacher qualification indicators and the socioeconomic level and student achievement. Therefore, teachers with more favorable characteristics would tend to work in schools with students with higher socioeconomics levels and better educational performance, and vice-versa. In terms of literature review, international studies investigating teacher mobility inside the same school system stand out. This research covers teachers joining the Rio de Janeiro City school system between 2002 and 2012 and investigates the relation between teacher turnover in schools and the main features of teachers and students. The analyses are based on the local legislation on the transfer of such educational professionals from a school to another; on teacher functional data and school transfer records provided by the Rio de Janeiro Department of Education (SME-RJ); in addition, data from Teste Brasil (a natiowide test), regarding socioeconomic info and educational achievement of students. Major results include a possible convergence between poorer profiles of teachers and students in schools with higher teachers's turnover.

\section{Keywords}

Teachers' turnover - Non-random distribution of teachers among schools - Convergences between teachers and student profiles.

\section{Introdução}

A qualidade do ensino costuma ser diretamente associada à qualificação dos professores, sendo esse pensamento recorrente tanto entre estudiosos da área quanto entre aqueles que não são especialistas no assunto. Portanto, pode-se dizer que a importância do papel do professor é uma unanimidade.

$\mathrm{Na}$ academia não é diferente. Alguns estudos buscam, inclusive, identificar os fatores associados ao sucesso desses profissionais, traçando perfis docentes relacionados a melhores e/ou piores resultados de desempenho escolar, destacando características específicas, tais como os anos de experiência em sala de aula, a formação acadêmica, o domínio do conteúdo e de aspectos didáticos (CLOTFELTER et al., 2007a; RIVKIN et al., 2005; ROCKOFF, 2004, 2010).

Autores brasileiros que se dedicam a estudar o tema destacam a importância do investimento em políticas para a melhoria da formação dos professores e das condições 
de trabalho existentes nas escolas públicas. Esses dois fatores têm sido diretamente relacionados à qualidade da educação (ALMEIDA, 2004; GATTI; BARRETO, 2009; BRANDÃO; CARVALHO, 2015).

Além das características individuais dos professores, investiga-se também a importância de aspectos relacionados à equipe docente como um todo, principalmente no que diz respeito à coesão do grupo. A ausência de tal conexão tende a ser associada à falta de estabilidade na equipe, que costuma ter como consequência dificuldades para a consolidação de um planejamento pedagógico e o baixo desempenho dos alunos.

Com o objetivo de explicar as razões que levam à ausência de estabilidade do corpo docente ou à alta rotatividade de professores, como tem sido tratado este tema, muitos estudos buscam compreender como ocorre a distribuição de docentes entre escolas de uma mesma rede de ensino, as quais formalmente deveriam ter os mesmos critérios de ingresso, transferência e saída de professores.

0 enfoque deste estudo consiste justamente em investigar os aspectos associados à alta rotatividade docente, tendo como objeto de pesquisa as escolas públicas da Rede Municipal de Ensino do Rio de Janeiro.

A bibliografia utilizada é predominantemente internacional, composta principalmente por estudos norte-americanos e ingleses, pois esses exploram de maneira sistemática os padrões de rotatividade de professores em suas redes de ensino, com base em dados abrangentes que fornecem um panorama sobre determinada realidade. Os estudos nacionais a respeito do tema ainda são exploratórios neste sentido.

Dentre as referências utilizadas, grande parte dos resultados indica que a distribuição de professores entre instituições de ensino não ocorre de forma neutra. Destaca-se a existência de tendências que se repetem em relação ao perfil de professores e alunos de uma mesma escola, especificamente no que diz respeito à qualificação docente, ao nível socioeconômico dos alunos e ao desempenho escolar desses. Ou seja: professores mais bem qualificados tendem a lecionar em escolas que têm alunos com nível socioeconômico mais alto e melhor desempenho escolar, e vice-versa (BASCOPÉ; MECKES, 2010; BOYD et al., 2005, 2008, 2009; CLOTFELTER et al., 2006; GREENBERG; MACCALL, 1974; RUFFINELLI; GUERRERO, 2009; SCAFIDI et al., 2005; SMITHERS; ROBINSON, 2005; TOLEDO et al., 2010).

$\mathrm{Na}$ medida em que são verificadas as referidas convergências entre características docentes e discentes, estudos constatam que, assim como esperado, as unidades escolares mais afetadas costumam ser as de nível socioeconômico mais baixo, justamente aquelas que tendem a apresentar piores indicadores de desempenho escolar. Assim, tal desvantagem consiste principalmente na tendência de essas instituições receberem maior quantitativo de professores menos qualificados. Desta forma, as escolas que deveriam ser foco de maiores investimentos são as mais suscetiveis aos referidos mecanismos de mobilidade. Essa lógica incita questionamentos a respeito de aspectos relacionados à equidade entre escolas, no que se refere ao acesso às mesmas condições de aprendizagem, principalmente em relação a instituições públicas de ensino de uma mesma rede.

Portanto, com inserção na referida temática, este artigo trata das dinâmicas de rotatividade docente na rede municipal de ensino da cidade do Rio de Janeiro, tendo como pressuposto a existência de padrões não aleatórios de distribuição de professores entre escolas. Sugere-se que haja convergências entre perfis docentes e discentes nas 
instituições de ensino, em relação à qualificação docente, ao nível socioeconômico dos alunos e ao desempenho escolar. Supõe-se que essa configuração, do ponto de vista macro, produza e cristalize contextos de desigualdade de oportunidades educacionais dentro da própria rede.

\section{O que dizem as pesquisas acerca dos fatores associados à rotatividade docente nas escolas}

A existência de rotatividade de professores nas escolas não é considerada um problema em si, visto que todas as instituições de ensino convivem com esse fato em certa proporção. No entanto, Allensworth; Poniscia; Mazzeo (2009) ponderam que, enquanto a existência de alguma rotatividade é normal e esperada, altas taxas podem produzir problemas organizacionais, como a impossibilidade de consolidar o trabalho em equipe entre professores e a interrupção do planejamento pedagógico realizado durante o ano letivo, podendo gerar consequências negativas para o processo de aprendizagem dos alunos.

Sendo assim, a maior parte dos estudos que aborda essa problemática investiga principalmente os fatores que levam determinadas escolas a perderem maior quantitativo de professores e aqueles que fazem com que outras instituições sejam mais bem-sucedidas em manterem os docentes de sua equipe. Essa abordagem é importante para a compreensão do fenômeno da rotatividade docente nas escolas, já que a captação e a permanência de professores são questões centrais do ponto de vista das instituições, pois afetam diretamente o trabalho pedagógico e o cotidiano escolar, principalmente quando se trata de escolas que atendem alunos com condições financeiras menos favoráveis (BERRY; HIRSCH, 2005; DEE, 2004; GUARINO et al., 2006).

Dentre os estudos que investigam os aspectos associados à saída dos professores das escolas, muitos têm evidenciado que apenas pequena parcela dessa perda se refere à aposentadoria. A respeito de tal constatação, Ingersoll (2001) utiliza-se da metáfora revolving door (porta giratória) para se referir à existência de grande número de professores qualificados que deixam seus cargos precocemente.

A respeito dessa questão, a literatura esclarece que a existência de alta rotatividade docente em determinadas instituições de ensino pode estar associada a fatores referentes ao entorno destas, tais como acessibilidade, segurança e indicadores sociodemográficos. No entanto, grande parte das evidências de pesquisas acadêmicas sugere que, em geral, a mobilidade de professores entre escolas costuma seguir um sentido bem definido, na medida em que se constata a tendência de docentes mais bem qualificados lecionarem em escolas que possuem alunos com perfil mais favorável. Isso ocorre, possivelmente, devido à maior probabilidade de esses docentes preencherem as vagas nas referidas instituições de ensino, as quais tendem a ser mais atrativas, e, por outro lado, também mais seletivas (PODGURSKY et al., 2004 apud CABEZAS, 2010; CLOTFELTER et al., 2006b).

Greenberg e Maccall (1974) já contemplavam, na década de 1970, aspectos referentes à convergência entre o perfil dos alunos e dos professores na mesma instituição de ensino, de forma a buscar explicar a mobilidade docente entre escolas. Ao investigarem transferências de professores dentro de um mesmo distrito, os autores apontavam características não referentes ao salário, as quais denominaram "não econômicas", que 
seriam responsáveis por diferenciarem as escolas mais atrativas das menos atrativas na percepção dos docentes. Em meio a essas particularidades, destacavam-se o desempenho escolar e o nível socioeconômico dos alunos.

De acordo com essa dinâmica, professores de escolas que tivessem alunos com nível socioeconômico mais baixo e pior desempenho escolar tenderiam a mudar de escola, e docentes de escolas cujo alunado possuísse nível socioeconômico mais favorável e bom desempenho escolar permaneceriam e acumulariam experiência. Como resultado, os profissionais mais experientes lecionariam em escolas com alunos de nível socioeconômico mais alto e com melhor desempenho, enquanto professores mais jovens e menos experientes lecionariam em escolas com perfil oposto. Como consequência, as últimas escolas seriam também submetidas à maior rotatividade docente, devido à dificuldade em reter professores.

Estudos mais recentes evidenciam resultados semelhantes. Clotfelter et al. (2006b), por exemplo, destacam altos percentuais de rotatividade docente em escolas de nível socioeconômico mais baixo. Por meio de indicadores sociais do alunado, entre 1999 e 2004, os autores constataram que docentes tendem a se transferir para escolas de nível socioeconômico mais alto.

Os estudos nacionais acerca da temática ainda são escassos e exploratórios, no entanto, já encontramos resultados relevantes como os referentes à pesquisa realizada por Duarte (2009), a partir de dados do Sistema Nacional de Avaliação da Educação Básica (SAEB) de 2003. Dentre os indicadores mais consistentes destacam-se a relação entre o aumento de alunos brancos no $5^{\circ}$ ano do Ensino Fundamental e a redução da rotatividade de professores; a menor rotatividade docente na rede privada de ensino quando comparada à rede pública, no $5^{\circ}$ e no $9^{\circ}$ ano; e a maior rotatividade em escolas com maiores índices de violência, principalmente no $5^{\circ}$ ano do Ensino Fundamental. Outro exemplo de estudo realizado no Brasil, em São Miguel Paulista - São Paulo, é a pesquisa de Alves et al. (2014), com resultados que apontam escolas de entorno menos vulnerável e compostas por alunos com maior nível socioeconômico recebendo professores mais qualificados e experientes.

Além das evidências tratadas até aqui, a literatura que investiga o tema da rotatividade docente é elucidativa ao enfatizar que, se por um lado, verifica-se significativa convergência de perfis menos favoráveis de alunos e professores em uma mesma instituição de ensino, por outro lado, escolas com essas características tendem a oferecer piores condições de trabalho aos seus docentes (JOHNSSON et al., 2005). Portanto, embora muitos estudos destaquem o nível socioeconômico e o desempenho escolar dos alunos como fatores determinantes para a saída dos professores das escolas, trabalhos mais recentes mostram que a variação na mobilidade docente atribuída ao perfil dos alunos pode ser, na verdade, atribuída a fatores contextuais, ou seja, à ausência de condições de trabalho adequadas nas escolas (GRISSOM, 2011; JOHNSON et al., 2005; LADD, 2009; LOEB et al. 2005).

Podemos exemplificar essa constatação por meio da seguinte hipótese: estudantes de famílias de nível socioeconômico baixo e com pior desempenho escolar tenderiam a estudar em instituições de ensino com piores condições de trabalho, sendo os últimos aspectos os responsáveis pelas altas taxas de saída de professores destas escolas e não as características dos alunos em si. 
Esses resultados são de extrema importância, já que quando as características dos alunos são apontadas como os principais fatores de influência sobre as altas taxas de saída de professores das escolas, cria-se um grande impasse para a proposição de políticas públicas. Por outro lado, quando se constata relação estreita entre aspectos relativos às condições de trabalho e a rotatividade docente, tais elementos contextuais passam a ocupar posição central nas pesquisas, justamente por serem mais facilmente passíveis de mudança.

\section{Procedimentos metodológicos}

A amostra deste estudo abrange os docentes que lecionavam para o ensino fundamental regular das escolas públicas da rede municipal de ensino do Rio de Janeiro, entre os anos de 2002 e 2012. Este recorte de dez anos foi definido durante minha pesquisa de doutorado, realizada de 2011 a 2014. Faz parte da amostra um total de matrículas igual a 18.831. Ressalta-se aqui a possibilidade de determinado número de professores terem duas matrículas. Em relação ao recorte de escolas contemplado, registram-se 1042 instituições, sendo que todas têm ao menos um segmento do ensino fundamental e fazem parte da trajetória profissional dos referidos professores.

A principal base de dados do estudo foi fornecida pela Secretaria Municipal de Educação do Rio de Janeiro (SME-RJ), contendo informações funcionais dos docentes que ingressaram na Rede entre 2002 e 2012. Esse é o principal banco de dados, na medida em que contém os registros de transferências dos professores entre escolas.

De forma a complementar a referida base de dados, foram incorporadas informações da Prova Brasil de 2007 a 2011, que dizem respeito ao desempenho escolar e ao nível socioeconômico dos alunos das escolas da rede pública de ensino do município do Rio de Janeiro.

Abaixo estão listadas as variáveis utilizadas nas análises, com informações sobre como foram criadas.

Quadro 1 - Variáveis utilizadas no estudo

\begin{tabular}{|l|l:l|}
\hline \multicolumn{1}{|c|}{ Variável } & \multicolumn{1}{|c|}{ Tipo } & \multicolumn{1}{c|}{ Descrição } \\
\hline $\begin{array}{l}\text { Percentual de rotatividade docente } \\
\text { 2002-2012 }\end{array}$ & Contínua & N de transferências / No de matrículas que ingressaram entre 2002 e 2012 \\
\hline Professores recém-concursados & Contínua & Média de professores recém-concursados na rede \\
\hline Nível de formação & Ordinal & Ensino médio (magistério): 1; Ensino superior: 2; Pós-graduação: 3 \\
\hline Nível socioeconômico dos alunos & Ordinal & Alto: 1; Médio-alto: 2; Médio: 3; Médio-baixo: 3 \\
\hline Pós-graduação & Contínua & Média aritmética de professores com Pós-graduação \\
\hline Universidade pública & Contínua & Média aritmética de professores formados em universidade pública \\
\hline NSE médio & Contínua & Média aritmética do nível socioeconômico dos alunos \\
\hline $\begin{array}{l}\text { Desempenho abaixo do nível básico em } \\
\text { matemática }\end{array}$ & Contínua & Média aritmética dos alunos que tiveram desempenho abaixo do nível básico \\
\hline
\end{tabular}

Fonte: Elaboração própria. 
a) Percentual de rotatividade docente 2002-2012: pensada para atuar como proxy da rotatividade docente na rede municipal de ensino do Rio de Janeiro, consiste no número de transferências ocorridas sobre o total de professores ingressantes durante os dez anos que compreendem o recorte deste estudo.

b) Professores recém-concursados: professores com até três anos de exercício na rede municipal de ensino do Rio de Janeiro. Este dado é importante devido à dificuldade que os docentes encontram nos primeiros anos de magistério, o que pode influenciar na qualidade do trabalho que desempenham em sala de aula. Além disso, a revisão de literatura mostra a tendência de professores menos experientes lecionarem em escolas com maior rotatividade docente, as quais costumam ter alunos com nível socioeconômico mais baixo, pior desempenho escolar e condições de trabalho menos favoráveis. Isto ocorre devido ao fato de os professores mais experientes e capacitados ocuparem as vagas das escolas com as melhores condições gerais de trabalho e menor rotatividade.

c) Nível de formação: categorias referentes ao nível de formação dos professores: (1) Ensino médio, com especialização para o magistério; (2) Ensino superior; (3) Pósgraduação. 0 nível de formação foi utilizado nas análises por ser considerada uma das características dos professores que exercem influência sobre as dinâmicas de rotatividade docente. Esse dado também é tratado por meio de outra variável, que consiste na média de professores com pós-graduação, opção feita por ser o nível mais alto de formação, com o intuito de investigar se a existência de professores com esse perfil exerce alguma influência sobre a referida mobilidade nas escolas.

d) Desempenho abaixo do nível básico em matemática: o indicador de proficiência dos alunos adotado aqui consiste em um dos níveis de medição que embasaram a produção do IDESP - Índice de Desenvolvimento da Educação de São Paulo (SOARES, 2009). Estes são divididos em uma escala que geralmente é composta por quatro níveis: abaixo do básico, básico, adequado e avançado. Optou-se pela análise do nível de desempenho abaixo do básico em matemática, com o objetivo de investigar aspectos relacionados a alunos com resultados de desempenho abaixo da média. Quanto à disciplina investigada, a proficiência em matemática é considerada por muitos autores a medida mais exata para investigar o conhecimento propriamente escolar (SOARES, 2009). No estudo são considerados os resultados da Prova Brasil dos alunos do primeiro e do segundo segmento, ou seja, do $5^{\circ}$ e do $9^{\circ}$ ano. Foram analisados os dados de 2007, por se tratar do período intermediário do recorte adotado.

e) Nível socioeconômico dos alunos (NSE): média do nível socioeconômico dos alunos das escolas estudadas, do $5^{\circ}$ e do $9^{\circ}$ ano. Este dado também é tratado por meio de níveis, tais como: alto, médio-alto, médio e médio-baixo. A medida de NSE foi gerada pelo INEP, por meio de dados provenientes dos questionários contextuais da Prova Brasil, a partir da edição de 2011, sobre questões que dizem 
respeito à renda familiar, à posse de bens e contratação de serviços de empregados domésticos pela família dos estudantes, além do nível de escolaridade de seus pais ou responsáveis. A definição das questões que integrariam o indicador se deu por meio de uma análise criteriosa dos questionários contextuais. Após essa análise, 17 questões foram escolhidas e podem ser observadas no Quadro 2.

Quadro 2 - Codificação das questões que compõem o NSE

\begin{tabular}{|c|c|}
\hline Descrição & Questão \\
\hline TV por assinatura \\
\hline Nivel de rendimento da família & Rádio \\
\hline & Videocassete ou DVD \\
\hline & Geladeira \\
\hline Nível educacional dos pais & Freezer \\
\hline & Cóquina de lavar roupa \\
\hline & Computador \\
\hline & Benda familiar \\
\hline & Telefone fixo \\
\hline & Escofone celular \\
\hline & Espirador de pó \\
\hline
\end{tabular}

Fonte: Brasil (2013).

Esclarecidas algumas questões referentes às variáveis utilizadas nas análises, cabe destacar que este estudo teve como base a legislação que regulamenta as transferências de professores entre escolas. Para isto, foi feito um levantamento principalmente das resoluções da Secretaria Municipal de Educação do Rio de Janeiro (SME-RJ) a respeito das transferências entre escolas da rede municipal de ensino, bem como das portarias da Coordenadoria de Recursos Humanos da SME-RJ, que estabelecem normas para a concretização de tais movimentos.

0 primeiro levantamento de dados realizado diz respeito ao exame das normas que regem as transferências de professores entre escolas da referida rede de ensino, ao longo do período correspondente ao recorte da pesquisa, ou seja, entre 2002 e 2012. São analisados os regulamentos publicados pela Secretaria Municipal de Educação (SME-RJ), tendo como enfoque principal os critérios que deliberam sobre a possibilidade de mudança de escola.

No que diz respeito à análise dos dados quantitativos, primeiramente é fornecida uma visão geral acerca dos percentuais anuais de transferências, em relação ao total de matrículas de professores existentes em cada ano. Nesse sentido, uma mesma matrícula 
pode ser considerada mais de uma vez, caso tenham sido registradas mais de uma transferência relativa a esta, assim como pode haver, em contrapartida, matrículas não consideradas no cálculo, caso não apresentem registros de transferências.

A segunda etapa de análise consiste em verificar as dinâmicas de rotatividade docente nas instituições de ensino, tendo a escola como unidade de investigação. Em um primeiro momento, são expostos gráficos originados de cruzamentos entre indicadores de rotatividade docente e as principais características de professores e alunos. Em seguida, é apresentada uma regressão linear multivariada.

0 último método de análise referido no parágrafo anterior é possibilitado pelo programa Minitab. A regressão linear multivariada é uma operação estatística que permite verificar a existência de relação matemática entre $\mathrm{X}$ (variável(is) independente(s)) e Y (variável(is) dependente(s)). No que diz respeito a aspectos gráficos, a regressão busca a reta mais próxima dos pontos observados. Sua equação é igual a: $\mathrm{y}=\mathrm{a}+\mathrm{bx}$. 0 indicador de rotatividade utilizado é o percentual de rotatividade de professores, que consiste na chamada variável dependente. Dentre as variáveis independentes, destacam-se aspectos que dizem respeito ao perfil de professores e alunos, principalmente em relação à formação acadêmica dos professores, ao nível socioeconômico do alunado e ao desempenho escolar deste.

\section{Possibilidades de mobilidade de professores entre escolas na rede municipal do Rio de Janeiro, conforme legislação específica}

Os processos de ingresso, de saída e de transferência de professores entre escolas da rede municipal de ensino do Rio de Janeiro são regulamentados por legislação específica. Particularmente no que diz respeito às transferências de professores entre escolas, são tratadas a seguir, de forma breve, algumas normas estabelecidas por resoluções e portarias publicadas pela Secretaria Municipal de Educação do Rio de Janeiro (SME-RJ), e correspondentes ao recorte metodológico deste estudo, referente aos anos de 2002 a 2012. Estes dados foram obtidos através de consulta ao Sistema de Documentação, disponível no site da Secretaria Municipal de Educação do Rio de Janeiro.

0 provimento inicial dos cargos de professor na Rede Municipal de Ensino do Rio de Janeiro é realizado por meio de concurso público para o preenchimento de vagas nas escolas, as quais são distribuídas entre Coordenadorias Regionais de Educação (CREs), de acordo com suas localidades. No ato de inscrição para o concurso, o candidato opta pela Coordenadoria Regional de Educação (CRE) para a qual deseja concorrer, geralmente com base na proximidade entre as escolas que a compõem e sua residência. Caso seja aprovado, sua alocação se torna restrita a uma das escolas abrangidas pela coordenadoria de escolha: "Art.2 - Os candidatos inscrever-se-ão na CRE de sua escolha concorrendo, exclusivamente, apenas às vagas nela existentes” (RIO DE JANEIRO, 2002).

A referida lei também prevê que, para ter o direito a se transferir de uma escola para outra, é preciso que o professor permaneça na mesma instituição na qual ingressou por, pelo menos, cinco anos. Entretanto, apesar da aparente impossibilidade de transferência antes deste prazo, admite-se que tal regra possa ser flexibilizada de acordo com as especificidades da legislação em vigor, sendo algumas tratadas a seguir. 
A possibilidade de exceção se aplicava às regras para transferência de professores entre escolas da mesma Coordenadoria Regional de Educação (CRE), entre os anos de 2002 a 2009, na medida em que neste período se exigia o mínimo de três anos de permanência na primeira escola em que o professor ingressasse. Contudo, a partir de 2010, essa exigência passou a ser de cinco anos. No que se refere às transferências entre escolas de CREs diferentes, o período mínimo de permanência na instituição de ensino de origem já consistia em cinco anos, norma que se encontrava em vigor desde o ano de 2002.

A regulamentação do processo de transferência de escola se dá por meio da abertura dos chamados concursos de remoção, realizados anualmente. Nesses há critérios específicos de classificação, sendo atribuído a cada um deles um total de pontos que, somados, definem a colocação final dos candidatos. Ao analisar os referidos critérios, constata-se que o tempo de efetivo exercício no âmbito da rede municipal de ensino é um dos principais requisitos para que o professor seja mais bem colocado e consiga a transferência. Como exemplos são destacados a seguir trechos de resoluções da Secretaria Municipal de Educação do Rio de Janeiro em dois diferentes momentos:

Resolução SME N. ${ }^{\circ}$ 0820, de 30 de Janeiro de 2004:

Art. $1^{\circ} 0$ direito de preferência dos servidores para terem exercício na unidade escolar de origem obedecerá, sucessivamente, aos seguintes critérios:

I - tempo de serviço na Unidade Escolar;

II - tempo de serviço na Coordenadoria Regional de Educação;

III - tempo de serviço público municipal, no cargo;

IV - número de pontos de chegada à Unidade Escolar; quando for o caso;

V - menor número de ausências ao serviço.

Portaria E/SUBG/CRH/SME n. ${ }^{\circ}$ 03, de 14 de outubro de 2011:

Art. 6. ${ }^{\circ}$ Para efeito de cálculo dos pontos de que trata o art. $4^{\circ}$ da Resolução SME n. ${ }^{\circ} 1163$, de 13 de outubro de $2011^{2}$, levar-se-á em consideração o tempo de efetivo exercício, em dias, no cargo atual, contado a partir da data da posse até 31/08/2011.

Desta forma, embora nas escolas municipais do Rio de Janeiro os processos de transferência sejam regulados por normas específicas, essas tendem a beneficiar os professores mais experientes, já que lhes garantem a preferência no direito de escolha da escola para a qual desejam se transferir. Assim, na medida em que os professores mais experientes escolhem em primeiro lugar, a tendência é que busquem escolas com melhores condições gerais de trabalho. Portanto, esta dinâmica pode gerar uma distribuição desigual de professores entre escolas, tal como mostrado pela literatura.

2- Art. $4 .{ }^{\circ}$ Ao candidato será atribuído um total de pontos, observados os critérios estabelecidos pela Coordenadoria de Recursos Humanos. Parágrafo único. A remoção atenderá, rigorosamente, à classificação dos candidatos, em ordem decrescente de pontos. 
Essa mesma circunstância pode ser verificada em outras redes de ensino do país, como é o caso do município de São Miguel Paulista, no Estado de São Paulo, no qual as transferências de professores tendem a ocorrer de escolas localizadas em bairros mais vulneráveis em termos sociais e culturais para escolas situadas em bairros menos vulneráveis, de acordo com a escolha dos docentes, com base em critérios de antiguidade (ALVES et al., 2014).

A respeito dessa questão, Donaldson (2011) pondera que decisões de alocação do corpo docente não podem ser limitadas por critérios específicos, tais como políticas de antiguidade, as quais contribuem para aumentar a desigualdade de distribuição de professores entre escolas, no que se refere à qualificação destes.

\section{Mobilidade de professores entre escolas da Rede Municipal de Ensino do Rio de Janeiro - de 2002 a 2012}

Por meio de dados fornecidos pela Secretaria Municipal de Educação da cidade do Rio de Janeiro, este trabalho apresenta um levantamento inicial acerca da mobilidade de professores entre escolas em um período de dez anos, entre 2002 e 2012. Objetiva-se verificar elementos que permitam um entendimento inicial acerca da dimensão dessa mobilidade. Tem-se como intuito verificar de que modo esse fenômeno afeta a referida rede de ensino.

Os dados abaixo evidenciam totais absolutos e percentuais de transferência de matrículas docentes entre escolas. As análises foram realizadas com base no número de matrículas, e não no número de docentes que tomaram posse, já que um professor pode ter mais de uma matrícula na rede. Além disso, as análises consideraram apenas aquelas que ingressaram na rede municipal entre os anos de 2002 e 2012, visto que não foi possível ter acesso aos dados de movimentos de matrículas ingressantes nos anos anteriores.

Tabela 1 - Quantitativo de transferências entre escolas e de saídas do sistema de ensino por ano

\begin{tabular}{|c|c|c|c|c|c|c|}
\hline Ano & $\begin{array}{c}\text { Total de transferências } \\
\text { de escola }\end{array}$ & $\begin{array}{l}\text { Total de } \\
\text { Posses }\end{array}$ & $\begin{array}{c}\text { Matrículas que } \\
\text { saíram do Sistema }\end{array}$ & $\begin{array}{c}\text { Frequência } \\
\text { acumulada de } \\
\text { matrículas }\end{array}$ & $\begin{array}{l}\text { Transferências de } \\
\text { Escola por ano (\%) }\end{array}$ & $\begin{array}{l}\text { Saídas do Sistema } \\
\text { por ano (\%) }\end{array}$ \\
\hline 2002 & 524 & 1764 & 5 & 1764 & $29,71 \%$ & $0,28 \%$ \\
\hline 2003 & 1877 & 3791 & 3 & 5550 & $33,82 \%$ & $0,05 \%$ \\
\hline 2004 & 1753 & 170 & 7 & 5717 & $30,66 \%$ & $0,12 \%$ \\
\hline 2005 & 2489 & 766 & 7 & 6476 & $38,43 \%$ & $0,11 \%$ \\
\hline 2006 & 1552 & 248 & 18 & 6717 & $23,11 \%$ & $0,27 \%$ \\
\hline 2007 & 1604 & 698 & 35 & 7397 & $21,68 \%$ & $0,47 \%$ \\
\hline 2008 & 1979 & 1377 & 58 & 8739 & $22,65 \%$ & $0,66 \%$ \\
\hline 2009 & 2806 & 1523 & 50 & 10204 & $27,50 \%$ & $0,49 \%$ \\
\hline 2010 & 2719 & 2472 & 87 & 12626 & $21,53 \%$ & $0,69 \%$ \\
\hline 2011 & 3534 & 4081 & 146 & 16620 & $21,26 \%$ & $0,88 \%$ \\
\hline 2012 & 3764 & 1941 & 173 & 18415 & $20,44 \%$ & $0,94 \%$ \\
\hline
\end{tabular}

Fonte: Secretaria Municipal de Educação do Rio de Janeiro (SME-RJ), 2002-2012. Elaboração própria. 
A tabela acima fornece, para cada ano, uma visão geral acerca dos totais absolutos e dos percentuais de transferências de professores entre escolas, além de saídas da rede municipal de ensino do Rio de Janeiro. Os percentuais de mudança de escola foram gerados a partir dos totais absolutos de mudança por ano sobre a frequência acumulada de matrículas. Já os percentuais de saída do sistema de ensino foram gerados por meio do número de saídas registrado por ano, sobre a frequência acumulada de matrículas.

A penúltima coluna evidencia os percentuais de transferências de escola por ano. Acerca desse dado, não se trata de percentuais de matrículas sobre as quais foram registradas transferências de escola, já que pode haver tanto matrículas sem registros de transferência quanto aquelas com mais de um registro. Portanto, é possível falar apenas em percentuais de mudanças de escola por ano, em relação ao total de matrículas consideradas naquele período.

A partir de uma primeira observação, é possível verificar percentuais próximos de movimentos de professores entre escolas, comparados os anos de 2002 a 2012, sendo verificada uma variação de $20 \%$ a 38\%. No que diz respeito aos percentuais de saídas do sistema por ano, constatam-se baixas taxas, as quais alcançam no máximo 0,94\%.

\section{Rotatividade docente nas escolas e o perfil de professores e alunos}

São expostas abaixo análises descritivas e uma regressão linear, com abordagem de indicadores de rotatividade docente nas instituições de ensino e características específicas de professores e alunos. A princípio, serão expostas as análises descritivas e, em seguida, a regressão linear.

Dentre os indicadores de rotatividade abordados destacam-se as seguintes variáveis: percentual de rotatividade de professores e média de professores recém-concursados. Em relação ao primeiro indicador citado, o objetivo é utilizá-lo como uma espécie de proxy da rotatividade de professores nas escolas. No que se refere aos professores recém-concursados, pretende-se verificar se a maior proporção deles está associada a características menos favoráveis da instituição de ensino, tal como aponta a literatura, quando mostra maior quantitativo de professores inexperientes em escolas com maior oferta de vagas, visto que determinadas instituições de ensino possuem dificuldades para captar professores, fato que pode estar relacionado a piores condições de trabalho oferecidas aos docentes (PODGURSKY et al., 2004, apud CABEZAS, 2010; CLOTFELTER et al., 2006b, 2007a; DARLING-HAMMOND; SYKES, 2003; LOEB et al., 2005; RIBEIRO 2013).

Os gráficos de 1 a 3 mostram resultados de análises descritivas bivariadas, com base em cruzamentos entre variáveis referentes à rotatividade docente e a características dos professores.

0 gráfico 1 indica diferenças percentuais discretas, que mostram a tendência de escolas com menor nível de formação docente apresentarem maior percentual de rotatividade de professores, sendo 56\% em instituições de ensino cuja maioria dos docentes tem formação de nível médio, referente à especialização em magistério, o antigo curso normal. Com resultados convergentes aos obtidos aqui, muitos estudos mostram maior rotatividade docente em escolas que têm professores com piores indicadores de formação (BOYD et al., 2009; CLOTFELTER et al., 2011; FIGLIO, 2002; HANUSHEK et al., 2004; SCAFIDI et al., 2005). 
Gráfico 1 - Percentual de rotatividade docente nas escolas, por nível de formação dos professores

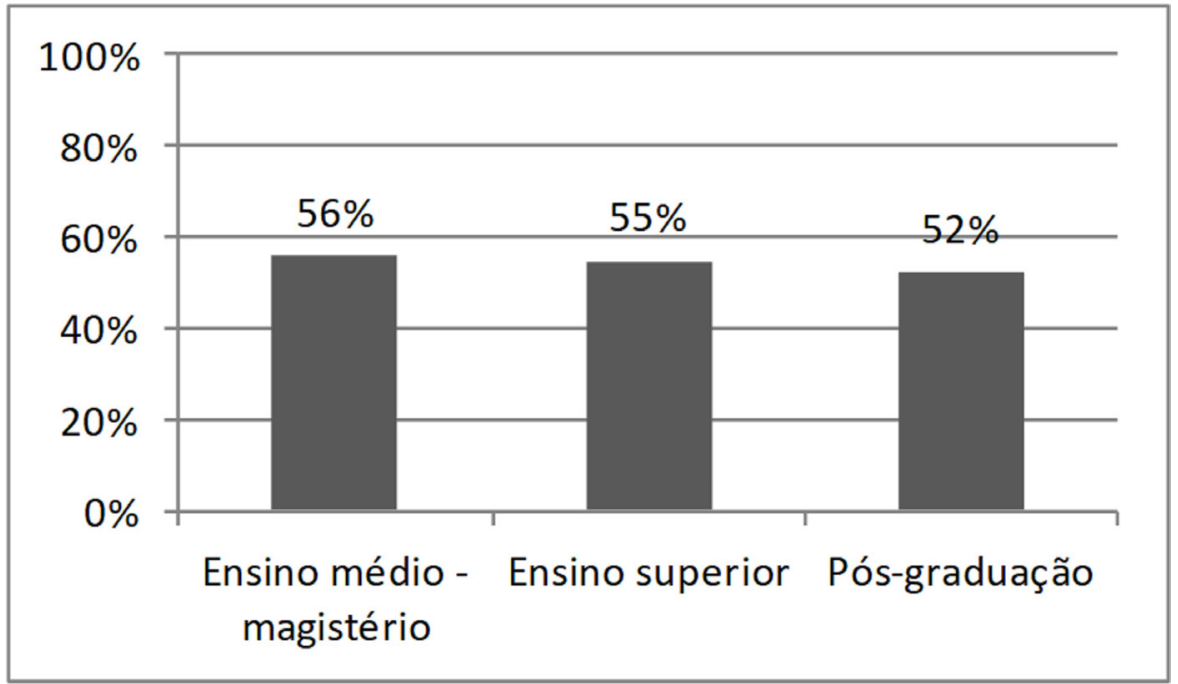

Valor-P (significância) do gráfico 1: 0,000.

Fonte: Elaboração própria.

Gráfico 2 - Percentual de rotatividade docente nas escolas, por nível socioeconômico dos alunos

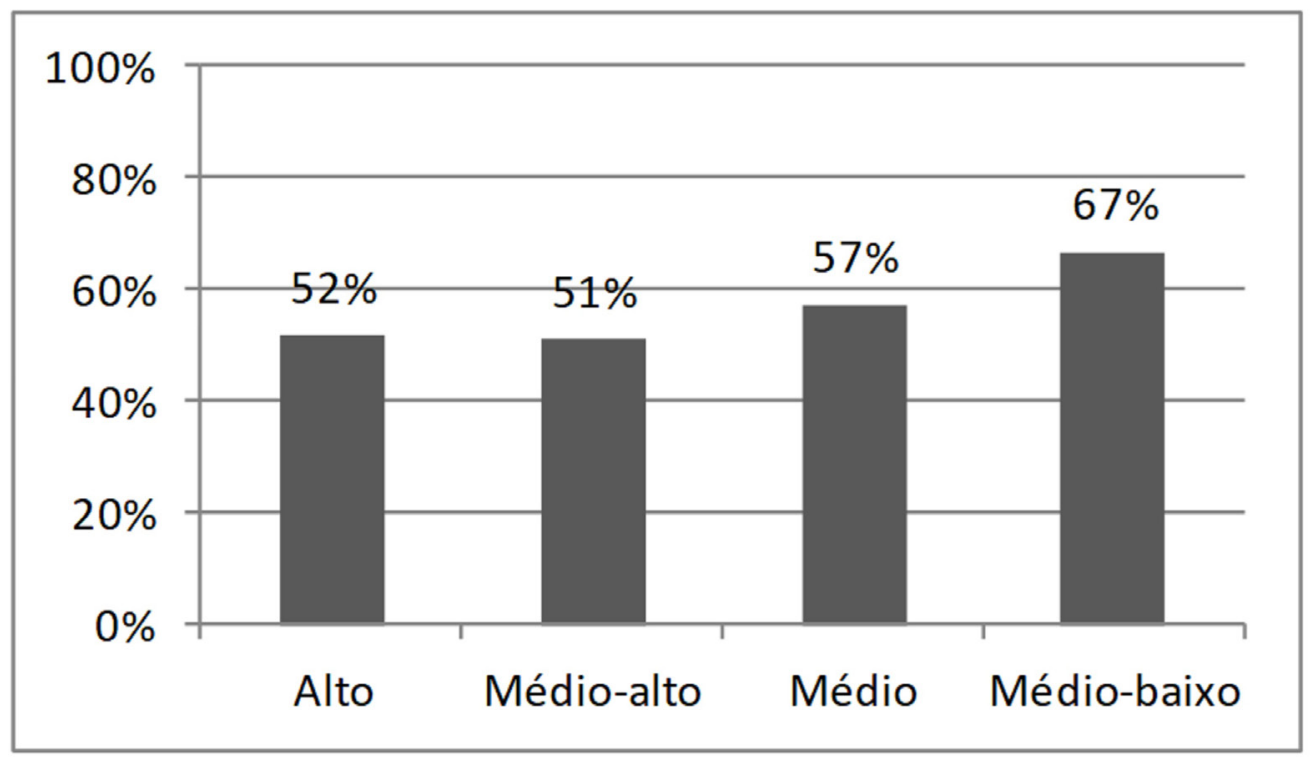

Valor-P (significância) do gráfico 2: 0,000.

Fonte: Elaboração própria. 
Já em relação ao nível socioeconômico dos alunos, o gráfico 2 expõe percentuais mais altos de rotatividade docente em escolas que possuem nível socioeconômico mais baixo. Verifica-se, desta forma, que instituições de ensino com alunos de nível socioeconômico médio-baixo apresentam percentuais de rotatividade em torno de $67 \%$. Constata-se novamente correspondência entre os dados acima e os resultados divulgados por estudos de referência, na medida em que inúmeras pesquisas evidenciam taxas mais altas de rotatividade docente em escolas que possuem alunado com esta característica (ALLENSWORTH; PONISCIA; MAZZEO, 2009; BASCOPÉ; MECKES, 2010; CLOTFELTER et al., 2006b; RUFFINELLI; GUERRERO, 2009).

As análises abaixo destacam o cruzamento entre o nível socioeconômico dos estudantes e um indicador de ausência de experiência docente, relativo ao percentual de professores recém-chegados à rede de ensino.

Gráfico 3 - Percentual de professores recém-concursados, por média de nível socioeconômico dos alunos nas escolas

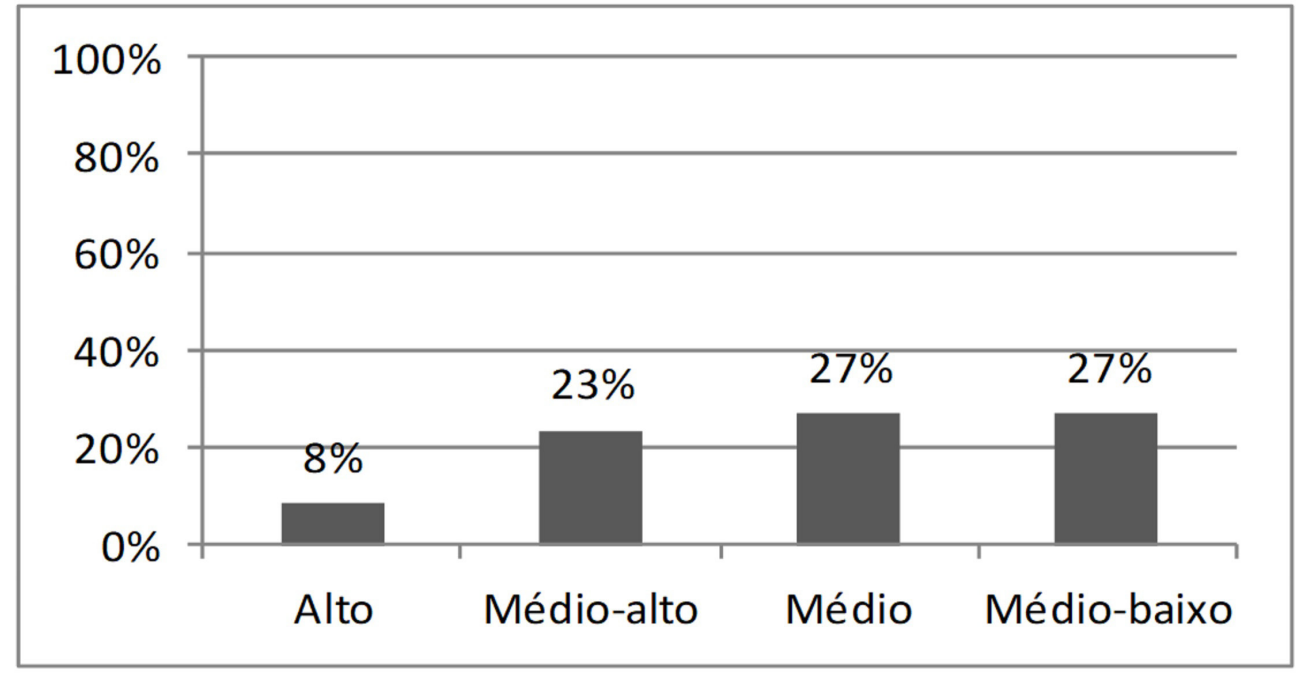

Valor-P (significância) do gráfico 3: 0,000.

Fonte: Elaboração própria.

A partir do gráfico acima é possível verificar que escolas com alunos de nível socioeconômico mais baixo recebem maior percentual de professores recém-concursados. Esse resultado corresponde à hipótese de que professores menos experientes tendem a ser alocados em escolas compostas por um alunado de nível socioeconômico mais baixo, as quais também possuem piores condições de trabalho, conforme destacado pela literatura (PODGURSKY et al., 2004 apud CABEZAS, 2010; CLOTFELTER et al., 2006b, 2007a; DARLING-HAMMOND; SYKES, 2003; LOEB et al., 2005; RIBEIRO 2013; ALVES et al., 2014).

No que diz respeito aos aspectos das escolas apontados como mais atrativos aos professores, assim como já evidenciado, o perfil dos alunos destaca-se como um dos fatores mais abordados pela literatura, principalmente no que se refere ao nível socioeconômico e 
ao desempenho escolar, fatores de atração que têm sido atualmente bastante relacionados às condições de trabalho nas escolas (CLOTFELTER et al., 2006b; GREENBERG; MACCALL, 1974; HANUSHEK; RIVKIN, 2006; JACKSON, 2009; REED; RUEBEN; BARBOUR, 2006; TOLEDO et al., 2010). As análises subsequentes buscam justamente verificar em que medida essa tendência se confirma, em relação às referidas características do alunado.

\section{Análise multivariada sobre indicador de rotatividade docente, perfil de professores e alunos}

Nesta seção, será realizada uma regressão linear simples, de forma a aprofundar a análise de alguns aspectos destacados até então. No que diz respeito à unidade de análise, optou-se por verificar a rotatividade docente nas escolas, visando a investigar a relação entre este aspecto e características de professores e alunos, tais como indicadores de qualificação docente, nível socioeconômico e desempenho escolar dos estudantes.

0 emprego de um indicador de rotatividade de professores como variável dependente tem como intuito investigar fatores relacionados a esse fenômeno. As variáveis independentes utilizadas buscam principalmente contemplar características dos professores referentes à qualificação docente e a características dos alunos relativas ao nível socioeconômico e ao desempenho escolar. Os indicadores de qualificação docente são: título de pós-graduação e formação acadêmica em universidade pública. Esta última variável é considerada indicador de qualificação docente devido não só ao prestígio que possuem as universidades públicas, como também aos resultados de exames nacionais que medem o desempenho dos alunos destas instituições, que as colocam em patamar superior quando comparadas às instituições privadas. Considera-se ainda o fato de o desenvolvimento da pesquisa no Brasil estar concentrado nas universidades públicas.

Quanto às características dos alunos, são abordados o nível socioeconômico e o desempenho escolar, especificamente um indicador que corresponde ao nível de desempenho abaixo do básico em matemática, na Prova Brasil de 2007. Assim como em relação ao perfil dos professores, as características dos alunos contempladas aqui se justificam pela ampla abordagem em estudos sobre o tema, sendo frequentemente associadas às dinâmicas de rotatividade docente.

Tabela 2 - Regressão Linear para percentual de rotatividade de professores do ensino fundamental

\begin{tabular}{lcccc}
\hline & Variáveis & Coef & Valor-P & VIF \\
\hline Constante & 112,16 & 0,000 & \\
\hline Universidade Pública* & $-6,51$ & 0,012 & 1,13 \\
\hline Pós Graduaçaõ $^{* *}$ & $-25,45$ & 0,000 & 1,12 \\
\hline NSE Médio** & $-9,93$ & 0,000 & 1,34 \\
\hline Desemp. abaixo do básico - Matemática & 6,21 & 0,099 & 1,32 \\
\hline
\end{tabular}

Variável dependente: Percentual de rotatividade docente nas escolas;

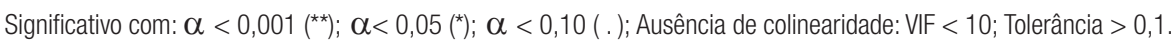

Fonte: Elaboração própria. 


\section{Poder de explicação do modelo de regressão $0^{3}$}

\begin{tabular}{|ll|}
\hline R2 & R2(aj) \\
$13,33 \%$ & $12,86 \%$ \\
\hline
\end{tabular}

Todas as variáveis apresentam coeficientes significativos. Dentre as que têm sinal negativo, destacam-se: formação em universidade pública, pós-graduação e nível socioeconômico dos alunos. A variável que indica a posse de título de pós-graduação se destaca por ter coeficiente mais alto, seguida pelo nível socioeconômico dos alunos e pela formação em universidade pública. Assim, é possível sugerir que escolas com maior percentual de rotatividade de professores costumam ter também menor quantitativo de professores com pós-graduação, alunos de nível socioeconômico mais baixo e menor quantitativo de professores formados em universidade pública.

Com sinal positivo, destaca-se a variável: desempenho abaixo do nível básico em matemática. A partir deste dado é possível supor que escolas com maior rotatividade docente possam apresentar também maior quantitativo de alunos com nível de desempenho abaixo do básico em matemática.

Conforme já citado durante a revisão bibliográfica, estudos abordam a convergência entre perfis do corpo docente e discente (BOYD et al., 2009; CLOTFELTER et al., 2006b; GREENBERG; MACCALL, 1974; SCAFIDI et al., 2005; SMITHERS; ROBINSON, 2005). A referida hipótese pode ajudar a explicar alguns dos resultados observados aqui, no que diz respeito à associação entre perfil menos favorável de professores, em termos de formação acadêmica, e de alunos com nível socioeconômico mais baixo e pior desempenho escolar, sendo tais convergências verificadas em instituições de ensino que possuem maior rotatividade docente. No entanto, esses resultados devem ser interpretados com cautela, devido ao baixo poder explicativo da regressão analisada. Da mesma forma devem ser tratadas as demais análises expostas na seção anterior, devido ao caráter descritivo destas.

\section{Considerações finais}

Os resultados destacados são convergentes às evidências verificadas pela literatura e às hipóteses levantadas por este estudo, especialmente em relação ao pressuposto de que a distribuição de professores entre escolas da rede municipal de ensino do Rio de Janeiro esteja associada a processos não aleatórios. Dessa forma, as constatações centrais expostas aqui se referem à possibilidade de haver perfis menos favoráveis de professores e alunos em instituições de ensino com maior rotatividade docente, em relação ao nível socioeconômico e desempenho escolar dos estudantes e à capacitação dos professores.

Em sua maioria, as referências bibliográfıcas utilizadas são internacionais, devido à ainda escassa literatura nacional acerca do tema. No entanto, alguns estudos realizados no país já apontam maior rotatividade docente e maior dificuldade de algumas escolas atraírem professores, de acordo com características de suas localidades e conforme o perfil do alunado (DUARTE, 2009; RIBEIRO, 2013, ALVES et al., 2014).

3- As variáveis independentes utilizadas na regressão linear explicam em torno de $13 \%$ da rotatividade docente (variável dependente). 
Além da referida tendência, em alguns estudos internacionais evidencia-se que a rotatividade docente associada ao perfil dos alunos pode ser, na verdade, atribuída às más condições de trabalho nas escolas (JOHNSON, 2005). Nesse sentido, determinadas instituições de ensino podem reunir diversas situações desfavoráveis, em termos de professores menos capacitados e pouco experientes, alunos com menor nível socioeconômico e mais baixo desempenho escolar, além de piores condições de trabalhos oferecidas aos docentes.

Considerando que as escolas com alunado de menor nível socioeconômico, desempenho escolar mais baixo e piores condições de trabalho deveriam ser foco de políticas públicas específicas, a lógica que se apresenta aqui é inversa. Isso porque os mecanismos de transferência de professores na Rede Municipal de Ensino do Rio de Janeiro, assim como mostrados neste estudo, são baseados em critérios de antiguidade. Ou seja: professores com mais tempo de exercício do magistério na rede, ao optarem por mudar de escola, têm prioridade na escolha da instituição para qual pretendem ir. Dessa forma, a referida dinâmica pode segregar cada vez mais instituições de ensino menos atrativas em relação às características citadas.

Esse dado é preocupante e pode ser estendido para outros contextos, uma vez que critérios de antiguidade, comumente utilizados no serviço público para remoção de servidores, ao serem utilizados como regra para remoção de professores entre escolas de redes públicas, sem as devidas ponderações e sem considerar determinadas especificidades, podem gerar o efeito perverso descrito. Um exemplo de circunstância similar à tratada neste trabalho é o estudo de Alves et al. (2014), realizado no município de São Miguel Paulista, Estado de São Paulo, que indica transferências de professores de escolas localizadas em bairros mais vulneráveis em relação a aspectos sociais e culturais para escolas situadas em bairros menos vulneráveis, processo que ocorre também com base em critérios de antiguidade.

Tais resultados representam um alerta no que se refere à distribuição de professores entre escolas das redes públicas de ensino, que pode estar gerando conjunturas que beneficiam determinadas instituições em detrimento de outras, reproduzindo contextos de desigualdade de oportunidades educacionais dentro da própria rede.

Uma alternativa para a quebra desse círculo vicioso seria o investimento em políticas públicas destinadas a escolas mais vulneráveis em relação aos aspectos citados, e que sejam fundamentadas em ações conjugadas, visando não somente a atração de professores mais bem qualificados, mas especialmente a permanência destes. Isso pode se dar principalmente através da melhoria das condições de trabalho, aspecto que, como mencionado anteriormente, tem sido considerado elemento-chave por estudos recentes para entender o fenômeno da rotatividade docente associado ao perfil do alunado.

Por fim, cabe ressaltar o caráter exploratório deste estudo. 0 predomínio de análises de caráter descritivo justifica-se principalmente pela intenção de fornecer um primeiro panorama a respeito das dinâmicas de rotatividade docente em escolas da rede municipal do Rio de Janeiro, considerando a escassez de estudos nacionais sobre o tema com enfoque na investigação de redes públicas de ensino. 


\section{Referências}

ALLENSWORTH, Elaine; PONISCIA, Stephen; MAZZEO, Christopher. The schools teachers leave: teacher mobility in Chicago Public Schools. Chicago: Consortium on Chicago School Research: University of Chicago; 2009.

ALMEIDA, Maria Izabel. Docentes para uma educação de qualidade: uma questão de desenvolvimento profissional. Educar, Curitiba, n. 24, p. 165-176, 2004.

ALVES, Liciana et al. Remoção de professores e desigualdades em territórios vulneráveis. Cadernos Cenpec, São Paulo, v. 4, n. 2, p. 122-145, dez. 2014.

BASCOPÉ, Martin; MECKES, Lorena. Distribución inequitativa de los nuevos profesores mejor preparados: "Características de origen y destino laboral de los egresados de pedagogía básica". In: CONGRESO INTERDISCIPLINARIO DE INVESTIGACIÓN EN EDUCACIÓN BOLETÍN, 1., 2010, Santiago de Chile. 30 de setembro de 2010. Primer... Santiago de Chile: [S. n.], 2010. Trabalho apresentado no evento.

BERRY, Barnett; HIRSCH, Eric. Recruiting and retaining teachers for hard-tostaff schools. Washington, DC: National Governors Association Center for Best Practices, 2005.

BOYD, Donald et al. Explaining the short careers of high-achieving teachers in schools with low-performing students. American Economic Review, Albany, v. 95, n. 2, 166-71, 2005. Disponível em: <http://web. stanford.edu/ sloeb/Short\%20Careers\%20\%282\%29.pdf>. Acesso em: 07 abr. 2019.

BOYD, Donald et al. The influence of school administrators on teacher retention decisions. National Bureau of Economic Research Working Paper, Cambridge. n. 25, May, 2009.

BOYD, Donald et al. Who leaves? Teacher attrition and student achievement. National Bureau of Economic Research Working Paper, Cambridge, n. 14022, May, 2008. Disponível em: <http://www.nber.org/papers/ w14022>. Acesso em: 07 abr. 2019.

BRANDÃO, Zaia; CARVALHO, Cynthia. Qualidade do ensino, balanço de uma década de pesquisas. Educação \& Sociedade, Campinas, v. 36, n. 131, p. 445-458, abr./jun. 2015.

BRASIL. Instituto Nacional de Estudos e Pesquisas Educacionais Anísio Teixeira. Ministério da Educação e Cultura. Diretoria de Avaliação da Educação Básica. Nota técnica: Indicador de Nível Socioeconômico das Escolas de Educação Básica (INSE). Brasília, DF: INEP, 2013.

CABEZAS, Verónica. Factores correlacionados con las trayectorias laborales de docentes en chile, con especial énfasis en sus atributos académicos. Santiago de Chile: Pontificia Universidad Católica de Chile, 2010. Proyecto Fonide, n. FS511082.

CLOTFELTER, Charles et al. Teacher-Student Matching and the Assessment of Teacher Effectiveness. National Bureau of Economic Research Working Paper, Cambridge. n. 11936; Jan. 2006. Disponível em: <http://www.nber.org/papers/w11936>. Acesso em: 07 abr. 2019. 
CLOTFELTER, Charles et al. High poverty schools and the distribution of teachers and principals. National Bureau of Economic Research Working Paper, Cambridge, Dec. 2006b. Duke University.

CLOTFELTER, Charles et al. How and why do teacher credentials matter for student achievement: high poverty schools and the distribution of teachers and principals. National Bureau of Economic Research Working Paper, n. 2, Mar. 2007a. Sanford Institute. Duke University. Disponível em: <http://www.caldercenter.org/ sites/default/files/1001058_Teacher_Credentials.pdf>. Acesso em: 07 abr. 2019

CLOTFELTER, Charles et al. Teacher mobility, school segregation, and pay-based policies to level the playing field. Education Finance and Policy, Cambridge, v. 6, n. 3, p. 399-438, 2011.

DARLING-HAMMOND, Linda; SYKES, Gary. Wanted, a national teacher supply policy for education: the right way to meet the "Highly Qualified Teacher" challenge. Education Policy Analysis Archives, Arizona, v. 11, n. 33, p. 1-55. 2003. Disponível em: <http://epaa.asu.edu/ojs/article/viewFile/261/387>. Acesso em: 07 abr. 2019.

DEE, Jay. Turnover intent in an urban community college: strategies for faculty retention. Community College Journal of Research and Practice, Philadelphia, v. 28, n. 7, 593-607; 2004.

DONALDSON, Graham. Teaching Scotland's future. Edinburgh: Scottish Government, 2011.

DUARTE, Rafael. Os determinantes da rotatividade dos professores no Brasil: uma análise com base nos dados do SAEB 2003. 2009. Dissertação (Mestrado em Economia Aplicada) - Faculdade de Economia, Administração e Contabilidade da Universidade de São Paulo, Ribeirão Preto, 2009.

FIGLIO, David; GETZLER, Lawrence. Accountability, ability and disability: gaming the system? National Bureau of Economic Research Working Paper, Cambridge, n. 9307, Oct. 2002. Disponível em: <http:// www.nber.org/papers/w9307>. Acesso em: 07 abr. 2019.

GATTI, Bernadete; BARRETO, Elba (Org). Professores do Brasil: impasses e Desafios. Brasília, DF: Unesco, 2009.

GREENBERG, David; McCALL, John. Teacher mobility and allocation. The Journal of Human Resources, Wisconsin, v. 9, n. 4, p. 480-502, 1974.

GRISSOM, Jason. Can good principals keep teachers in disadvantaged schools? Linking principal effectiveness to teacher satisfaction and turnover in hard-to-staff environments. Missouri: Harry S Truman School of Public Affairs: University of Missouri, 2011.

GUARINO, Cassandra; SANTIBANEZ, Lucrecia; DALEY, Glenn. Teacher recruitment and retention: a review of the recent empirical literature. Review of Educational Research, Washington, DC, v 76, n. 2, p.173-208, 2006.

HANUSHEK, Eric et al. Why public schools lose teachers. The Journal of Human Resources, Wisconsin, v. 39, n. 2, 326-354, 2004. 
HANUSHEK, Eric; STEVEN, Rivkin. Teacher quality: handbook of the economics of education. v. 2. Amsterdam, [s. n.], 2006. Disponível em: <http://www.procon.org/sourcefiles/hanushek-teacher-quality.pdf >. Acesso em: 08 abr. 2019.

INGERSOLL, Richard. Teacher turnover and teacher shortages: an organizational analysis. Pensylvania: University of Pensylvania, 2001.

JACKSON, Clement. Student demographics, teacher sorting, and teacher quality: evidence from the end of desegregation. Journal of Labor Economics, Chicago, v. 27, n. 2, p. 213-256, 2009. Disponível em: $<$ https://digitalcommons.ilr.cornell.edu/workingpapers/78/>. Acesso em: 08 abr. 2019.

JOHNSON, Susan et al. Who stays in teaching and why: a review of the literature on teacher retention. Harvard: [s. n.], 2005. The project on the next generation of teachers. Disponível em: <http://assets.aarp. org/www.aarp.org_/articles/NRTA/Harvard_report.pdf>. Acesso em: 08 abr. 2019.

LADD, Helen. Teachers' perceptions of their working conditions: how predictive of policy-relevant outcomes? Educational Evaluation and Policy Analysis, Thousand Oaks, v. 33, n. 2, p. 235-261, Dec. 2009.

LOEB, Susanna; DARLING-HAMMOND, Linda; LUCZAK, John. How teaching conditions predict teacher turnover in California schools. Peabody Journal of Education, Abingdon, v. 80, n. 3, p. 44-70, 2005.

REED, Deborah; RUEBEN, Kim; BARBOUR, Elisa. Retention of new teachers in California. Public Policy Institute of California; San Francisco, 2006. Disponível em: <http://www.ppic.org/content/pubs/report/ R_206DRR.pdf>. Acesso em: 08 abr. 2019.

RIBEIRO, Eduardo. Impactos educacionais das Unidades de Polícia Pacificadora: explorando efeitos sobre fluxos docentes. Revista Intratextos, Rio de Janeiro, v. 4, n. 1, p. 27-52, 2013. Disponível em: <http:// www.e-publicacoes.uerj.br/index.php/intratextos/article/viewFile/8561/7025>. Acesso em: 08 abr. 2019.

RIO DE JANEIRO. Lei n 3357-02. 3 de janeiro de 2002. Rio de Janeiro: Câmara Municipal, 2002. Disponível em: <http://cm-rio-de-janeiro.jusbrasil.com.br/legislacao/263588/lei-3357-02>. Acesso em: 08 abr. 2019.

RIO DE JANEIRO. Secretaria Municipal de Educação. Portaria n. ${ }^{\circ}$ 03, DE 14 de outubro de 2011. Estabelece normas para a realização do concurso de remoção INTERCRE do pessoal do quadro do magistério (Professor I e Professor II), para regência nas unidades escolares, no ano 2012. Rio de Janeiro: SME, 2011.

RIO DE JANEIRO. Secretaria Municipal de Educação. Resolução SME No 820 DE 30 de janeiro de 2004. Estabelece normas para a movimentação de pessoal no âmbito das coordenadorias regionais de educação, durante 0 ano letivo. Rio de Janeiro: SME, 2004.

RIVKIN, Steven; HANUSHEK, Eric; KAIN, John. Teachers, schools, and academic achievement. Econometrica, New Haven, v. 73, n. 2, p. 417-458, Mar. 2005. Disponível em: <http://www.econ.ucsb.edu/ jon/ Econ230C/HanushekRivkin.pdf>. Acesso em: 08 abr. 2019. 
ROCKOFF, Jonah. The impact of individual teachers on student achievement: Evidence from panel data. American Economic Review, Pittsburgh, v. 94, n. 2, p. 247-52, Mar. 2004. Disponível em: <https://www0. gsb.columbia.edu/faculty/jrockoff/rockoff_teachers_march_04.pdf> Acesso em: 08 abr. 2019.

RUFFINELLI, Andrea; GUERRER0, Alexis. Círculo de segmentación del sistema educativo chileno: destino laboral de los egresados de pedagogía en educación básica. Calidad em La Educación, Santiago de Chile, n. 31, dic. 2009.

SCAFIDI, Benjamin et al. Race, poverty, and teacher mobility. Economics of Education Review, Atlanta, v. 26, p. 145-159, Aug. 2005.

SECRETARIA Municipal de Educação do Rio de Janeiro (SME-RJ). Disponível em: <http://www0.rio.rj.gov. br/sme/destaques/documentacao.htm>. Acesso em: 08 abr. 2019.

SMITHERS, Alan; ROBINSON, Pamela. Teacher turnover, wastage and movements between schools. Research Report, New York, n. 640, 2005. Centre for Education and Employment Research. Department for Education and Skills. Disponível em: <http://dera.ioe.ac.uk/5625/1/RR640.pdf>. Acesso em: 08 abr. 2019 .

SOARES, José Francisco. Índice de desenvolvimento da educação de São Paulo - IDESP: bases metodológicas. São Paulo em Perspectiva, São Paulo, v. 23, n. 1, p. 29-41, jan./jun. 2009.

TOLEDO, Gabriela et al. Calidad docente y logro escolar: enfrentando el problema de ordenamiento no aleatorio entre características de profesores y alumnos. 2010. Dissertação (Mestrado em Economia) - Facultad de Economía y Negocios. Escuela de Postgrado Economía y Negocio. Universidad de Chile, Santiago de Chile, 2010.

Recebido em: 15.03 .2018

Revisões em: 07.08.2018

Aprovado em: 25.09.2018

Marcela Brandão Cunha é doutora em educação pela Pontifícia Universidade Católica do Rio de Janeiro (PUC Rio). Atualmente é técnica em assuntos educacionais no Instituto Federal de Educação, Ciência e Tecnologia do Rio de Janeiro (IFRJ) e coordenadora geral de programas e projetos de pesquisa da referida instituição. 